\title{
Research Progress of Olfactory Nerve Regeneration Mechanism and Olfactory Training
}

\author{
Bian $\mathrm{Hu}^{1,2}$, Jingyu Zhang $\mathbb{D}^{3}$, Mengdan Gong', Yongqin Deng', Yujie Cao', Yizhen Xiang', Dong Ye (D) \\ 'Department of Otorhinolaryngology-Head and Neck Surgery, Lihuili Hospital of Ningbo University, Ningbo, 315040, Zhejiang, People's Republic of \\ China; ${ }^{2}$ Department of Otorhinolaryngology-Head and Neck Surgery, Ninghai First Hospital, Ningbo, 315699, Zhejiang, People's Republic of China; \\ ${ }^{3}$ Shanghai Jiao Tong University, Shanghai, 200030, People's Republic of China \\ Correspondence: Dong Ye, Department of Otorhinolaryngology-Head and Neck Surgery, Lihuili Hospital of Ningbo University, Ningbo, 3I5040, \\ Zhejiang, People's Republic of China, Tel +86 I381986I213, Fax +86 574-87392232, Email yedong518@sina.com
}

\begin{abstract}
The olfactory nerve $(\mathrm{ON})$ is the only cranial nerve exposed to the external environment. Hence, it is susceptible to damage from head trauma, viral infection, inflammatory stimulation, and chemical toxins, which can lead to olfactory dysfunction. However, compared with all other cranial nerves, the $\mathrm{ON}$ is unique due to its inherent ability to regenerate. This characteristic provides a theoretical basis for treatment of olfactory dysfunction. Olfactory training (OT) is one of the main treatments for olfactory dysfunction. It is easy to apply and has few side-effects, and has been shown to be efficacious for patients with olfactory dysfunction of various causes. To further understand the application value of ON regeneration and OT on olfactory dysfunction, we review the research progress on the mechanism of ON regeneration and OT.
\end{abstract}

Keywords: olfaction, regeneration, olfactory training, olfactory dysfunction

\section{Introduction}

An epidemiological survey showed that the incidence of olfactory dysfunction is much higher than assumed previously. The estimated prevalence of hyposmia is up to $15 \%$ and functional anosmia is $5 \%$ in the general population. ${ }^{1}$ These disorders can result in malnutrition, food poisoning, weight loss, depression, and other disturbances. ${ }^{2}$

Odor receptors expressed in olfactory neuronal cilia within the olfactory epithelium detect odors first. Compared with all other cranial nerves, the olfactory nerve $(\mathrm{ON})$ is unique due to its inherent ability to regenerate, and likely regenerates throughout the lifetime of a human. ${ }^{3}$ Several therapeutic strategies have been proposed based on ON plasticity, but the validity of most of those regimens is uncertain. Therefore, exploring ON mechanisms and finding new and efficacious treatment of olfactory dysfunction is a rational approach.

Olfactory training (OT) can be undertaken readily and has few side-effects. The basic principle is for patients to smell different odors repeatedly and in an organized manner over a long period of time. ${ }^{4}$ OT is realistic treatment with great potential in patients with olfactory dysfunction. Several studies have shown that olfactory dysfunction due to infection of the upper respiratory tract, trauma, neurologic disorders, or idiopathic disorders can be improved by OT, particularly for enhancement of the ability to discriminate and identify odors. The exact mechanism by which OT improves olfactory function is not clear. However, it is believed to be associated with neuroplasticity of the olfactory system, which provides a new idea for treatment of olfactory dysfunction.

\section{Research Progress in the Mechanism of ON Regeneration Basal Cells: Precursor Cells of Olfactory Receptor Neurons (ORNs)}

Nascent ORNs are differentiated from basal cells to supplement aging and injured neurons, and then maintain the homeostasis of olfactory epithelial cells. Two populations of basal cells lie within the olfactory epithelium: horizontal basal cells (HBCs) and globose basal cells (GBCs). ${ }^{5}$ In health, HBCs are considered to be a "reservoir" of stem cells that 
remain dormant and they can be activated by direct and severe damage to the epithelium. Activated HBCs are mainly differentiated into GBCs, which then expand and differentiate into ORNs and other types of epithelial cells. ${ }^{6}$ GBCs are the main proliferative population in the olfactory epithelium and they are not only responsible for the self-renewal of ORNs, but are also the main cells for regeneration of ORNs after minor and selective injury. ${ }^{7}$

Labeling of thymidine analogs and mitosis-related proteins has shown that GBCs are the main proliferative population in the olfactory epithelium. ${ }^{8}$ GBCs can differentiate to almost all epithelial cell types, including ORNs, HBCs, sustentacular cells, and Bowman's glands/ducts cells. GBCs express different transcription factors during different stages of development and regeneration of the olfactory epithelium, and have different differentiation potential, mainly sex determining region Y-box 2 (Sox2), paired box 6 (Pax6), achaete-scute complex-like 1 (Ascl1), neurogenin 1 (Neurog1) and neuronal differentiation factor 1 (Neurod1), as shown in Figure 1 and Table 1. Sox2 is recognized as a stem cell transcription factor that is expressed in both GBCs and HBCs, and is required for the transition of cells from the activated state to a neuronal progenitor state. ${ }^{9}$ Leucine-rich repeat-containing G-protein coupled receptor 5 (Lgr5) is expressed exclusively in the GBCs of neonatal and adult mice. Lgr5 is the target molecule of Wingless-Int (Wnt) signaling pathway. Wnt signaling can promote the proliferation of Lgr5+ GBCs and regeneration of olfactory neurons. ${ }^{10,11} \mathrm{Li}$ et al used a strain a strain that destroyed the function of endogenous Lgr5 gene to characterize Sox2 expression in GBCs under normal conditions and in a lesion-regeneration model. They showed that a lack of Sox 2 expression reduced the proportion of natural ORNs significantly. ${ }^{12}$ During neurogenesis, Sox 2 can expand the number of neuronal precursors, whereas Pax6 accelerated neuronal differentiation. ${ }^{13}$ The expression of Ascl1 promotes GBCs differentiation to neurons. A study documented a reduction in the number of ORNs and absence of Notch homolog 1, translocation-associated

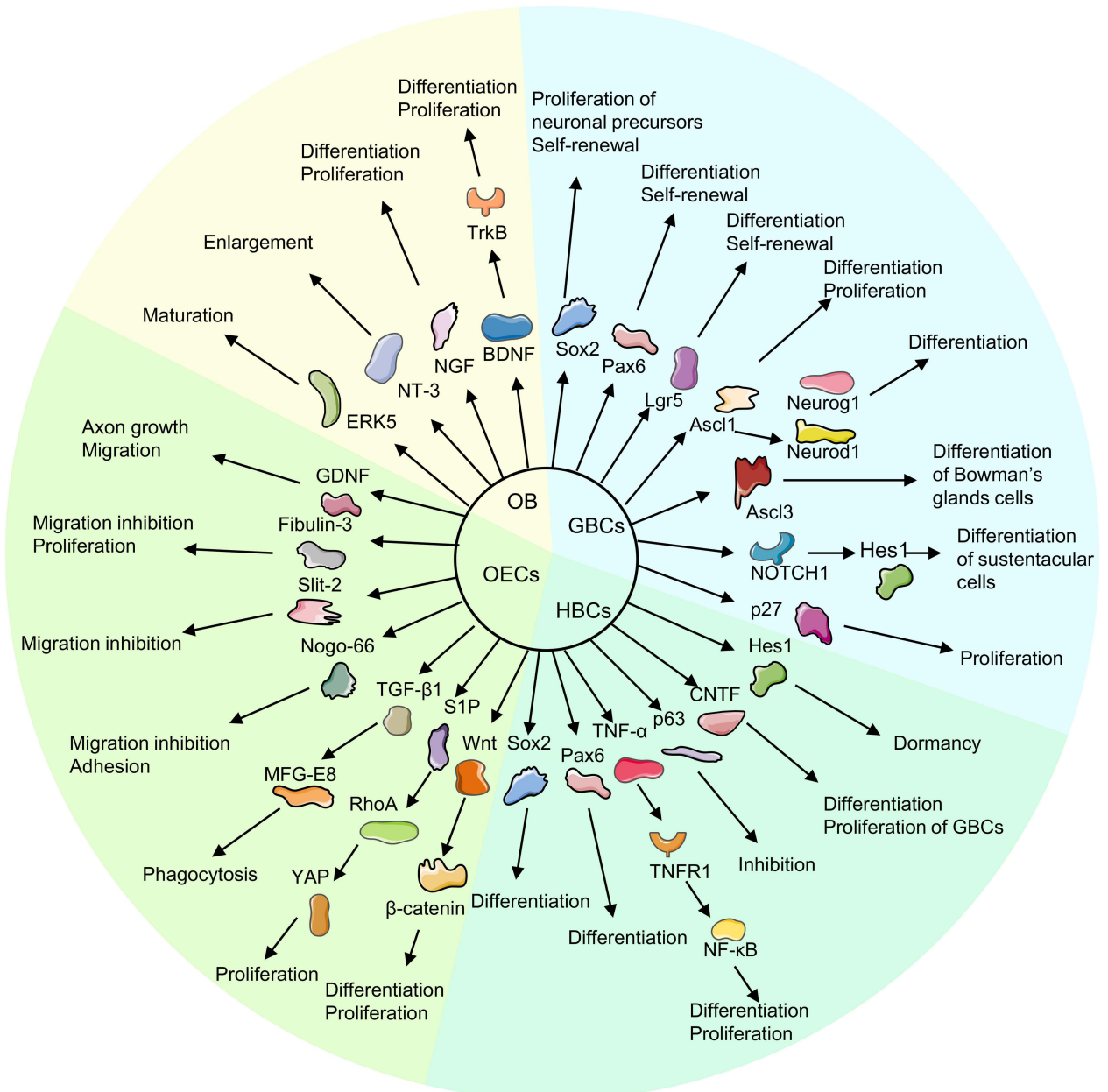

Figure I Mechanism of regeneration of the olfactory nerve. 
Table I Mechanism of Regeneration of the Olfactory Nerve

\begin{tabular}{|c|c|c|c|}
\hline Type & Regulator & Effects & References \\
\hline \multirow[t]{9}{*}{ GBCs } & Sox 2 & Proliferation of neuronal precursors, self-renewal & {$[9,12,13]$} \\
\hline & Pax6 & Differentiation, self-renewal & {$[13]$} \\
\hline & Lgr5 & Differentiation, self-renewal & {$[10,11]$} \\
\hline & NeurogI & Differentiation & {$[5]$} \\
\hline & NeurodI & Differentiation & {$[5]$} \\
\hline & Ascll & Differentiation, proliferation & {$[14]$} \\
\hline & Ascl3 & Differentiation of Bowman's glands cells & {$[47]$} \\
\hline & HesI & Differentiation of sustentacular cells & {$[14]$} \\
\hline & p27 & Proliferation & {$[8]$} \\
\hline \multirow[t]{6}{*}{ HBCs } & Sox2 & Differentiation & [9] \\
\hline & Pax6 & Differentiation & [9] \\
\hline & TNF- $\alpha$ & Differentiation, proliferation & {$[18]$} \\
\hline & CNTF & Differentiation, proliferation of GBCs & {$[20]$} \\
\hline & p63 & Inhibition & {$[22,24]$} \\
\hline & HesI & Dormancy & {$[24]$} \\
\hline \multirow[t]{7}{*}{ OECs } & GDNF & Axon growth, migration & [27-29] \\
\hline & Fibulin-3 & Migration inhibition, proliferation & [30] \\
\hline & Slit-2 & Migration inhibition & [48] \\
\hline & Nogo-66 & Migration inhibition, adhesion & [49] \\
\hline & TGF- $\beta$ I & Phagocytosis & [33] \\
\hline & SIP & Proliferation & [36] \\
\hline & Wnt & Differentiation, proliferation & [35] \\
\hline \multirow[t]{4}{*}{ OB } & BDNF & Differentiation, proliferation & {$[41,42]$} \\
\hline & NGF & Differentiation, proliferation & {$[40]$} \\
\hline & NT-3 & Enlargement & {$[43]$} \\
\hline & ERK5 & Maturation & {$[45]$} \\
\hline
\end{tabular}

Abbreviations: GBCs, globose basal cells; HBCs, horizontal basal cells; OECs, olfactory-ensheathing cells; OB, olfactory bulb; SOX2, sex determining region Y-box 2; Pax6, paired box; Lgr5, leucine-rich repeat-containing G-protein coupled receptor 5; Neurogl, neurogenin I; Neurod I, neuronal differentiation factor I; Ascl, achaete-scute complex-like; HesI, hairy and enhancer of split I; TNF$\alpha$, tumor necrosis factor- $\alpha$; CNTF, ciliary neurotrophic factor; GDNF, glial cell-derived neurotrophic factor; Slit-2, slit guidance ligand 2; Nogo-66, extracellular domain with 66-amino-acid-residue of Nogo protein; TGF- $\beta$ I, transforming growth factor- $\beta$ I; SIP, sphingosine Iphosphate; Wnt, Wingless-Int; BDNF, brain-derived neurotrophic factor; NGF, nerve growth factor; NT-3, neurotrophin 3; ERK5, extracellular regulated protein kinases 5.

(Drosophila) (NOTCH1)-expressing basal cells in achaete-scute complex-like 1 (Ascl1)-knockout mice, whereas NOTCH1 signaling suppressed Ascl1 via hairy and enhancer of split 1 (Hes1) and drove the differentiation of sustentacular cells, which showed mutuality between neurogenesis and sustentacular cells. ${ }^{14}$ Most GBCs express Ascl1 and become immediate neuronal precursor GBCs, followed by express Neurog1 and Neurod1 specifically, and 
finally differentiated into olfactory neurons. ${ }^{5}$ Immature neurons grow to the olfactory bulb (OB), thereby forming synaptic connections and differentiating into mature ORNs expressing olfactory marker proteins. ${ }^{15}$ This process can be reversed by damage to olfactory epithelium and ORNs. In the olfactory epithelium after olfactory bulbectomy and methimazole injury, Neurog1+ GBCs could express Sox2 and Pax6 and show multidirectional differentiation potential. Similarly, Ascl1+ GBCs resumed expression of Sox2 and Pax6 upon epithelial injury. Sox2 was required to initiate dedifferentiation and promote the expansion of multipotential progenitor cells, which suggested that the differentiation from stem cells to immediate precursor cells was not irreversible. ${ }^{16}$

HBCs remain largely in a quiescent state during normal neuronal turnover and even after an acute, selective loss of mature neurons. Iwai et al depleted mature ON selectively by excising the OB of mice. They found that HBCs differentiated predominantly into GBCs and, eventually, to ORNs after ON injury, but differentiated into Bowman's gland cells or sustentacular cells in a few cases. ${ }^{17}$ How HBCs are activated is not known. Studies have shown that expression of Sox 2 and Pax6 in HBCs is critical for neurogenesis after injury, and is associated with the maintenance of "stemness". In a mouse model of acute inflammation of the olfactory epithelium, proinflammatory cytokines (especially tumor necrosis factor (TNF)- $\alpha$ ) were involved in the proliferation and differentiation of HBCs through the tumor necrosis factor receptor 1/nuclear factor-kappa B (TNFR1/NF- $\mathrm{BB}$ ) pathway. Also, the acute (rather than the chronic) inflammatory state is more important in $\mathrm{ON}$ regeneration. ${ }^{18}$ Conversely, chronic inflammation was found to close down the regenerative function of HBCs to maintain epithelial immune defense. ${ }^{19}$ Jia et al instilled (i.n.) focal adhesion kinase in adult mice to promote ciliary neurotrophic factor (CNTF) expression by HBCs. They found that CNTF could affect GBCs in a paracrine manner, thereby promoting their proliferation and neuronal differentiation. ${ }^{20}$ Interestingly, recent studies have shown that HBC activation is often accompanied by downregulation of expression of the tumor protein p63. The latter is required for establishment of epithelial cells. ${ }^{21}$ P63 is highly enriched in HBCs. Injury stimulation caused downregulation of 63 expression, followed by differentiation of HBCs into various cell types such as GBCs. ${ }^{22}$ P63-knockout HBCs could strat differentiation without injury. ${ }^{23}$ In a lesion model (created by injury by the olfactotoxic gas methyl bromide), the downregulation of p63 expression activated HBCs to differentiate into GBCs. As the epithelium was gradually repaired, the expression of p63 also gradually increased, shifting HBCs from an activated to a quiescent state. These results indicate that the expression level of $\mathrm{p} 63$ is the main condition that determines whether HBC is activated or not. ${ }^{6}$ Herrick et al showed, by expression analysis, immunohistochemical examination, and next-generation sequencing, that the Notch signaling pathway maintained p63 expression and HBC dormancy. Jagged canonical Notch ligand 1 on sustentacular cells could drive downregulation of Notch pathway signaling after tissue injury to inhibit p63 expression and then activate $\mathrm{HBC}$ differentiation. ${ }^{24}$

\section{Olfactory-Ensheathing Cells (OECs): Unique Glial Cells}

OECs are the glial cells of the olfactory nervous system. They can support axons growth after injury and play an essential part in ON regeneration. ${ }^{25}$ During olfactory development, OECs arise from neural crest cells, ${ }^{26}$ extend ahead of pioneer olfactory axons, and cover the length of the entire ON from the peripheral nervous system into the central nervous system. Research has shown that each regenerating olfactory axon can follow the pathway created by pre-existing axons every 30-90 days. ${ }^{3}$ After ON injury, OECs maintain their structure and provide open channels for regenerating ON growth back to the OB. ${ }^{25}$ Studies have demonstrated that OECs can promote the regeneration of olfactory neurons, which is regulated by multiple mechanisms, including the production of neurotrophic factors, removal of neuron debris, the remyelination of damaged nerves.

Studies have shown that many neurotrophic factors can be secreted by OECs to promote neuronal migration and axon growth. OECs express and secrete glial cell-derived neurotrophic factor (GDNF) to promote axonal growth and neuronal survival, but also express GDNF receptors. ${ }^{27}$ GDNF could promote OECs migration by upregulating expression of GDNF family receptor- $\alpha 1$ and rearranged during transfection (Ret) receptors as well as activating c-Jun N-terminal kinase (JNK) signaling cascades. ${ }^{28}$ Windus et al observed the growth and interaction of olfactory axons and OECs using high resolution time-lapse microscopy. They suggested that GDNF could stimulate the lamellipodial waves of OECs, which resulted in the close cell-cell contact and migration of OECs, which increased the growth of olfactory axons. ${ }^{29}$ Real-time reverse transcription-quantitative polymerase chain reaction showed that OECs have high expression of the 
glycoprotein fibulin-3. Fibulin-3 could negatively regulate the ability of matrix metalloproteinase 2 to inhibit OECs migration and instead promote proliferation. These actions prevented ORN axons from entering other areas outside the $\mathrm{ON}^{30}$

OECs can phagocytose detrimental neuronal debris and create a favorable environment for ON regeneration. Nazareth et al utilized transgenic reporter lines in the developing olfactory system of mice to investigate phagocytosis initiation by OECs. They found that phagocytosis of olfactory axon debris by OECs was first detected at embryonic day-14.5 and demonstrated that OECs were the primary phagocytes of the ON in early embryonic development. ${ }^{31} \mathrm{Su}$ et al demonstrated that resting-state OECs could be activated by lipopolysaccharide stimulation to upregulate expression of phosphatidylserine receptors, which promoted the phagocytic ability of OECs. ${ }^{32}$ During phagocyte removal of neuron debris, OECs could enhance the phagocytic activity of OECs by regulating the integrin/milk fat globule epidermal growth factor 8 (MFG-E8) signaling pathway to secrete transforming growth factor (TGF)- $\beta 1 .^{33}$

Researchers transplanted OECs in transected ON from rats and observed recovery of the olfactory epithelium. Studies showed that, compared with the control group, the number of olfactory receptor neurons was greater in the OECtransplant group 2 weeks after surgery, ${ }^{34}$ which showed that OECs could remyelinate injured ON. Yue et al examined the Wnt pathway by immunostaining. They discovered that Wnt-activated OECs appeared after olfactory damage, and promoted the proliferation and differentiation of neurons through the $\mathrm{Wnt} / \beta$-catenin signaling pathway. ${ }^{35} \mathrm{~A}$ recent study showed that OECs could secrete sphingosine 1-phosphate (S1P), a sphingolipid metabolite with broad biological activities, and show high expression of S1P receptors which could bind with S1P, and then activate yes-associated protein (YAP) through the Ras homolog family member A (RhoA) pathway to promote OECs proliferation. These actions resulted in functioning of the S1P/RhoA/YAP pathway in ON growth. ${ }^{36}$

\section{OB: Target Organ of ON Development}

The OB receives axon terminals from primary olfactory neurons. ONs are considered to be mature upon establishment of synaptic connections with the mitral cells of the OB and expression of olfactory marker proteins. Therefore, the OB can be considered to be the target organ of the ONs. ${ }^{37,38}$ Kanaki et al co-cultured slices of the OB and blocks of the olfactory epithelium by a modified slice-culture method. They observed neuron-specific fibers extending to OB slices from olfactory-epithelium blocks after 4 days, ${ }^{39}$ which indicated that the OB had an inductive effect on ON growth in vitro.

Studies have shown that the OB can synthesize various trophic factors, such as nerve growth factor (NGF), brainderived neurotrophic factor (BDNF), GDNF, and interleukin-2, which travel retrogradely through the axon to the ON and maintain its survival. ${ }^{40-42}$ BDNF and neurotrophin 3 (NT-3) had no obvious influence on the number of neurons, but increased the length and branches of dendrites, which suggested that BDNF and NT-3 could stimulate neuronal maturation by increasing the size of synaptic boutons. ${ }^{43}$ Also, BDNF could regulate the development, proliferation, and projection of neurons in the central nervous system via tyrosine kinase receptor B (TrkB) signaling. ${ }^{44}$ Wang et al employed a site-specific, constitutively activated knock-in mouse line to activate endogenous extracellular regulated protein kinases (ERK5) specifically in OB regions of the brain. They found that ERK5 mitogen-activated protein kinase could enhance neuronal maturation in the OB and promote olfactory neurogenesis. ${ }^{45}$ Olfactory bulbectomy deprived the mature ON of the support of various trophic factors. This action caused ON death and then stimulated the proliferation and differentiation of basal cells, thereby improving ON regeneration. However, the regenerated olfactory epithelium in the olfactory-bulbectomy model was significantly thinner, indicating that the target organ was necessary for the survival and maturation of the $\mathrm{ON}^{46}$

The regeneration of $\mathrm{ON}$ is regulated by multiple mechanisms. The regulators and the mechanisms of $\mathrm{ON}$ regeneration in olfactory pathway are shown in Figure 1 and Table 1.

\section{Research Progress in OT OT Methods}

Studies have demonstrated that the classical OT method was developed on the basis of "odor prism" by Henning, and it is used widely in academia. This method requires participants to be exposed to four odors (phenyl ethyl alcohol (rose); 
eucalyptol (eucalyptus); citronellal (lemon); eugenol (cloves)) twice-daily for 12 weeks. In the morning and evening, participants are trained to sniff the odors for $\sim 10 \mathrm{~s}$. Odors are soaked in cotton pads and stored in four brown-glass jars labeled with the odor name to prevent spilling and for easy differentiation. ${ }^{4}$

With OT promotion, many researchers have undertaken studies on patients with olfactory dysfunction and made improvements on the classical OT method. The quantity, quality, and concentration of the odor, as well as the time and frequency of OT, influence the effect of OT to varying degrees. ${ }^{50-57}$ Some scholars prolonged the treatment time of OT from 12 weeks to 56 weeks, and found that long-term OT appeared to have a better therapeutic effect than short-term OT. ${ }^{52-54}$ Damm et al divided participants into two groups (high-training and low-training) according to the odor concentration. They discovered that participants in the high-training group were more likely to have improved olfactory function. ${ }^{56}$ Oleszkiewicz et al increased the frequency of OT to four times a day and, by contrast, OT twice-daily was more beneficial for olfactory recovery; this result might be associated with a "ceiling effect". ${ }^{57}$ Altundag et al changed the training odors periodically using 12 different odors to stimulate more and different olfactory receptors. ${ }^{51}$ Otherwise, Saatci et al designed an "olfactory training ball" with four odor-containing tubes to make OT simpler for improving the treatment compliance of participants. ${ }^{58}$

\section{Effect of OT Upon Olfactory Dysfunction}

OT has been demonstrated to enhance olfactory function in healthy people of all ages as well as in patients with olfactory dysfunction due to various causes (eg, nasal diseases, head trauma, infection of the upper respiratory tract, and neurodegenerative disease). A study showed that after a 12-week period of OT in healthy children that exposed to 4 mixtures of odors, children in the training group not only improved their sensitivity to the trained odors, but also had a significant improvement of general olfactory sensitivity compared with that in control-group children. ${ }^{59} 6$ weeks of OT in 8 -year-old children enhanced their ability of odor identification, but not odor sensitivity. The authors concluded that the main reason for the difference with the results of the previous studies was the age difference of the participants, as the OB volume and olfactory ability both increased with age. ${ }^{60}$ In a study of patients aged $>45$ years with olfactory disorders, the researchers used a modified form of OT with 12 odors and found that nearly half of 29 participants showed improvements in their odor threshold, discrimination and identification. ${ }^{61}$ In another study of the elderly, participants in the OT group had improved olfactory function, improved verbal function and subjective wellbeing, and a reduction of depressive symptoms, ${ }^{62}$ which improved their quality of life.

Studies have demonstrated that olfactory function primarily increases olfactory identification, followed by olfactory discrimination. ${ }^{63}$ In addition, a higher concentration of odors can improve olfactory function better than a low concentration of odors, and this effect seems to be particularly potent in patients with a disease course $<12$ months. ${ }^{56}$ A meta-analysis was employed to explore the effect of OT on postviral olfactory dysfunction. The authors showed clinically significant improvements in postviral olfactory dysfunction associated with OT. ${ }^{64}$ Choi et al used five odors (rose, cinnamon, orange, lemon, and peach) to carry out OT in patients with post-infection olfactory dysfunction: the OT group had better olfactory results with regard to the identification of and sensitivity to odors. ${ }^{65}$

A meta-analysis reviewed 812 abstracts and 13 full-text articles to evaluate the effects of OT on patients with posttraumatic olfactory disorders. The data indicated that OT could improve the ability of discrimination and identification of odors in patients with post-traumatic olfactory disorders within 8 months. ${ }^{66}$ After 6 months of OT, odor sensitivity could be improved slightly but the ability of odor identification remained unchanged. ${ }^{67}$ The authors postulated that the reasons for these inconsistent results might be due to differences in OT and olfactory-testing methods between institutions.

A prospective controlled non-blinded study on 70 patients with Parkinson's disease (PD) showed that after 12 weeks of OT, patients had significantly higher olfactory function, while those who did not undertake OT had unchanged olfactory function, which suggested that OT could help PD patients improve their olfactory function. ${ }^{68}$

\section{OT Mechanism in Olfactory Recovery}

\section{Peripheral Neuroplasticity: "Bottom-Up" Theory}

Odor receptors expressed in olfactory neuronal cilia within the olfactory epithelium detect odors first. These receptors belong to the superfamily of $\mathrm{G}$ protein-coupled receptors. The combination of odors and odor receptors triggers an 
electrical signal and transmits along the axons to the main $\mathrm{OB}$, and then the signal is transmitted to other regions of the brain. ${ }^{2}$ Different types of odors can stimulate different olfactory receptors and projection areas in OT, thus enhancing olfactory function. Studies have shown increased electro-olfactogram responses in patients receiving repeated exposure to odors. Hence, odourant-specific plasticity may start at the level of the olfactory epithelium. ${ }^{69} \mathrm{Kim}$ et al used an anosmia model in mice and found that mRNA expression of olfactory marker protein, guanine nucleotide-binding protein G(olf) subunit alpha (GNAL), and adenylyl cyclase (ADCY)-3 was higher in the olfactory neuroepithelium of OT mice than that in the olfactory neuroepithelium of no-treatment mice. During the early recovery period, the enzymatic activity of GNAL and ADCY seemed to be increased first in the OT group, suggesting that olfactory receptors were stimulated early. When OT was performed, the increase of glial fibrillary acidic protein (GFAP), an intermediate filament expressed in astrocytes, could stimulate the production or activity of OECs. Otherwise, an increase in expression of neurotrophic factors, such as BDNF and nerve growth factor receptor (NGFR), suggested that OT might stimulate the olfactory nervous system in a step-by-step approach. ${ }^{70}$ Recent studies showed that biomarkers such as $\mathrm{p} 75$ neurotrophin receptor (P75NTR), O4 antibody, smooth muscle alpha-actin (SMA) and soluble protein-100 (S-100) could be used to localize OECs. ${ }^{71,72}$ Compared with the hyposmia group and corticosteroid-treated group, mRNA expression of SMA and S-100 in the OT group was increased significantly after 3 weeks of treatment. ${ }^{73}$ Marin et al revealed that after OT, the number of migrating neuroblasts and proliferation of neural precursor cells were increased in bilateral OB damaged animals, which aided the recovery of olfactory function. ${ }^{74}$ Those findings suggested that OT may promote the proliferation and activity of OECs and neural precursor cells to stimulate olfactory regeneration.

Hasegawa et al undertook unilateral naris closures on mice. They observed that odor deprivation could lead to a loss of ORN activity and atrophy of the OB, both of which recovered upon odor stimulation. Otherwise, in the abatement of nasal inflammation without odor input, ORNs recovered partially, but a significant recovery of OB atrophy was not observed. Only with odor input could the OB recover completely from atrophy. The authors indicated that odor stimulation was necessary for the formation of OB neurons, ${ }^{75}$ which showed a bottom-up process of olfactory recovery.

\section{Central Mechanism: “Top-Down” Process}

The structure of many brain regions (especially the OB) changes after OT. The OB receives newly generated cells originating from the subventricular zone and maintains its plasticity. Studies have shown that the OB volume can be increased significantly in patients with olfactory dysfunction and healthy people who undertake OT, and olfactory function is also improved relatively, which reflects the neural plasticity of the olfactory system. Veyseller et al observed a decreased $\mathrm{OB}$ volume in patients after laryngectomy. ${ }^{76} \mathrm{~A}$ recent study found that the OB volume could be increased significantly in patients undergoing total laryngectomy after 6 months of OT. ${ }^{77}$ Negoias et al measured the OB volume before and after OT by magnetic resonance imaging of 97 healthy participants who undertook OT while closing the other nostril. They demonstrated that the OB volume increased also for untrained nostrils showing, for the first time, that OT could modulate the OB volume by a central mechanism. ${ }^{78}$

Studies have shown a reduction in gray matter (GM) volume in brain regions involved in olfactory-related processes, such as the orbitofrontal cortex, insular cortex, entorhinal cortex, piriform cortex, cingulate cortex, and amygdala, in patients with olfactory dysfunction. ${ }^{79-81}$ Han et al examined the changes in GM volume in patients with idiopathic olfactory disorders using a longitudinal approach. They found that patients who undertook OT had a larger GM volume in the medial orbitofrontal cortex and improved olfactory identification. ${ }^{82} \mathrm{Al}$ Ain et al evaluated 36 healthy young people and measured their brain-tissue density and cortical thickness using magnetic resonance imaging. They found that the thickness in the right entorhinal cortex, right inferior frontal gyrus, and bilateral fusiform gyrus of those who underwent OT was increased compared with that of healthy controls. ${ }^{83}$ Gellrich et al used voxel-based morphometry to compare changes in the OB volume and GM structure before and after OT in olfactory-dysfunction patients and healthy controls. Their data suggested that OT was related to an increased GM volume in the thalamus and hippocampus. ${ }^{84}$ Hence, OT might have psychological effects via the direct neural connections between the amygdala-hippocampal complex and ON.

In addition to structural changes, functional reorganization of the olfactory-related brain region may occur in patients with olfactory dysfunction. ${ }^{85-87}$ The piriform cortex receives an input from OB projection neurons and then projects it to higher olfactory areas (a brain structure with high involvement in olfactory perception). Kollndorfer et al observed that 


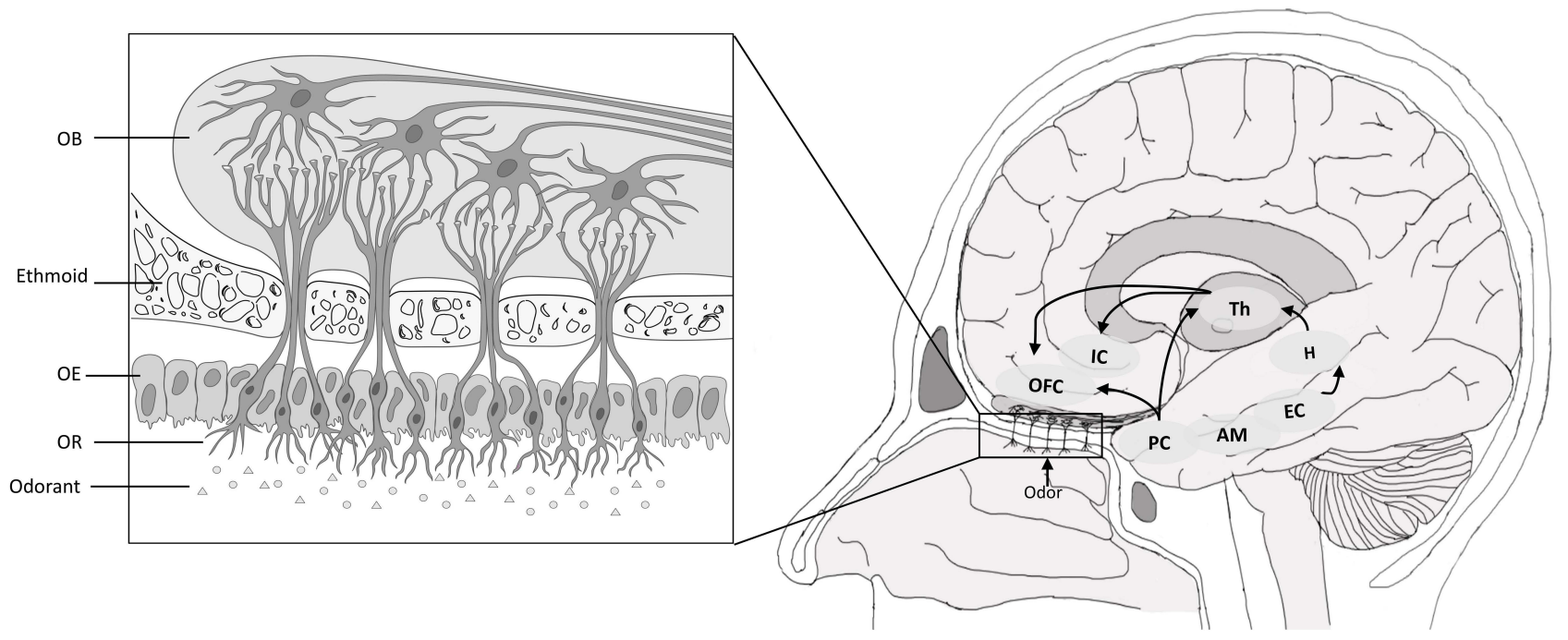

Figure 2 Mechanism of olfactory training in olfactory recovery.

Notes: I. OT increases the activity of olfactory receptors and secretion of various neurotrophic factors to promote the proliferation and activation of neural precursor cells stimulates regeneration of the olfactory system in a stepwise manner. 2. OT stimulates the central nervous system and leads to an increase in the gray-matter volume and functional reorganization of olfactory-related brain regions, including the OB, piriform cortex (PC), amygdala (AM), insular cortex (IC), orbitofrontal cortex (OFC), entorhinal cortex $(E C)$, hippocampus $(\mathrm{H})$ and the thalamus $(T h)$.

Abbreviations: $\mathrm{OB}$, olfactory bulb; $\mathrm{OE}$, olfactory epithelium; OR, olfactory receptors.

the piriform cortex in anosmic patients had abnormal networks with nonolfactory regions whereas, after OT, these nonolfactory functional connections declined, thereby suggesting that OT might promote neural reorganization. ${ }^{86}$

OT can promote olfactory regeneration through the peripheral system and central system. The mechanism is shown in Figure 2.

\section{Summary and Prospects}

The ON can be plastic and regenerative. Recent works on ON regeneration have shown some breakthroughs, but many areas remain unclear (eg, HBCs remain dormant in normal or slightly injured olfactory epithelium, however, after severe damage HBCs alone are unable to restore all cell types and instead lead to respiratory epithelial metaplasia. The extent to which injury to olfactory progenitor cells leads to functional decline and how it, in turn, leads to an imbalance in microenvironmental homeostasis and reduces the ability of $\mathrm{ON}$ regeneration). Clinical management of olfactory dysfunction remains a challenge. Some researchers have modified the duration, materials, and methods of OT to improve its pertinence and efficacy. Nevertheless, more research is needed to clarify the optimal odor concentration, training duration, frequency, and the most suitable population for OT.

\section{Acknowledgments}

This work was supported by grants from the Natural Science Foundation of Zhejiang Province (grant nos. LY19H160014, LY20H130001, LQ21H130001), Medical and Health Research Project of Zhejiang Province (grant nos. 2019ZD018, 2021KY307), Ningbo Health Branding Subject Fund (grant no. PPXK2018-02), Ningbo Natural Science Foundation (grant no. 202003N4239), the Ningbo "Technology Innovation 2025" Major Special Project (grant nos. 2018B10015, 2020Z097), and the Key Project of Teaching and Research of Ningbo University (grant no. JYXMXZD2021033).

\section{Disclosure}

The authors report no conflicts of interest in this work. 


\section{References}

1. Vennemann MM, Hummel T, Berger K. The association between smoking and smell and taste impairment in the general population. $J$ Neurol. 2008;255(8):1121-1126. doi:10.1007/s00415-008-0807-9

2. Glezer I, Malnic B. Olfactory receptor function. Handb Clin Neurol. 2019;164:67-78.

3. Yu CR, Wu Y. Regeneration and rewiring of rodent olfactory sensory neurons. Exp Neurol. 2017;287(Pt 3):395-408. doi:10.1016/j. expneurol.2016.06.001

4. Hummel T, Rissom K, Reden J, Hähner A, Weidenbecher M, Hüttenbrink K-B. Effects of olfactory training in patients with olfactory loss. Laryngoscope. 2009;119(3):496-499. doi:10.1002/lary.20101

5. Schwob JE, Jang W, Holbrook EH, et al. Stem and progenitor cells of the mammalian olfactory epithelium: taking poietic license. J Comp Neurol. 2017;525(4):1034-1054. doi:10.1002/cne.24105

6. Schnittke N, Herrick DB, Lin B, et al. Transcription factor p63 controls the reserve status but not the stemness of horizontal basal cells in the olfactory epithelium. Proc Natl Acad Sci U S A. 2015;112(36):E5068-E5077. doi:10.1073/pnas.1512272112

7. Leung CT, Coulombe PA, Reed RR. Contribution of olfactory neural stem cells to tissue maintenance and regeneration. Nat Neurosci. 2007;10 (6):720-726. doi:10.1038/nn1882

8. Jang W, Chen X, Flis D, Harris M, Schwob JE. Label-retaining, quiescent globose basal cells are found in the olfactory epithelium. J Comp Neurol. 2014;522(4):731-749. doi:10.1002/cne.23470

9. Gadye L, Das D, Sanchez MA, et al. Injury activates transient olfactory stem cell states with diverse lineage capacities. Cell Stem Cell. 2017;21 (6):775-790 e779. doi:10.1016/j.stem.2017.10.014

10. Chen M, Tian S, Yang X, Lane AP, Reed RR, Liu H. Wnt-responsive Lgr5 $5^{+}$globose basal cells function as multipotent olfactory epithelium progenitor cells. $J$ Neurosci. 2014;34(24):8268-8276. doi:10.1523/JNEUROSCI.0240-14.2014

11. Li X, Tong M, Wang L, Qin Y, Yu H, Yu Y. Age-dependent activation and neuronal differentiation of Lgr5+ basal cells in injured olfactory epithelium via notch signaling pathway. Front Aging Neurosci. 2020;12:602688. doi:10.3389/fnagi.2020.602688

12. Li Z, Wei M, Shen W, Kulaga H, Chen M, Lane AP. Sox2 regulates globose basal cell regeneration in the olfactory epithelium. Int Forum Allergy Rhinol. 2022. doi: $10.1002 /$ alr. 22890

13. Packard AI, Lin B, Schwob JE. Sox2 and Pax6 play counteracting roles in regulating neurogenesis within the murine olfactory epithelium. PLoS One. 2016;11(5):e0155167. doi:10.1371/journal.pone.0155167

14. Krolewski RC, Packard A, Jang W, Wildner H, Schwob JE. Ascl1 (Mash1) knockout perturbs differentiation of nonneuronal cells in olfactory epithelium. PLoS One. 2012;7(12):e51737. doi:10.1371/journal.pone.0051737

15. Rodriguez-Gil DJ, Bartel DL, Jaspers AW, Mobley AS, Imamura F, Greer CA. Odorant receptors regulate the final glomerular coalescence of olfactory sensory neuron axons. Proc Natl Acad Sci U S A. 2015;112(18):5821-5826. doi:10.1073/pnas.1417955112

16. Lin B, Coleman JH, Peterson JN, et al. Injury induces endogenous reprogramming and dedifferentiation of neuronal progenitors to multipotency. Cell Stem Cell. 2017;21(6):761-774 e765. doi:10.1016/j.stem.2017.09.008

17. Iwai N, Zhou Z, Roop DR, Behringer RR. Horizontal basal cells are multipotent progenitors in normal and injured adult olfactory epithelium. Stem Cells. 2008;26(5):1298-1306. doi:10.1634/stemcells.2007-0891

18. Chen M, Reed RR, Lane AP. Acute inflammation regulates neuroregeneration through the NF-kB pathway in olfactory epithelium. Proc Natl Acad Sci U S A. 2017;114(30):8089-8094. doi:10.1073/pnas.1620664114

19. Chen M, Reed RR, Lane AP. Chronic inflammation directs an olfactory stem cell functional switch from neuroregeneration to immune defense. Cell Stem Cell. 2019;25(4):501-513 e505. doi:10.1016/j.stem.2019.08.011

20. Jia C, Oliver J, Gilmer D, Lovins C, Rodriguez-Gil DJ, Hagg T. Inhibition of focal adhesion kinase increases adult olfactory stem cell self-renewal and neuroregeneration through ciliary neurotrophic factor. Stem Cell Res. 2020;49:102061. doi:10.1016/j.scr.2020.102061

21. Yoh K, Prywes R. Pathway regulation of p63, a director of epithelial cell fate. Front Endocrinol. 2015;6:51. doi:10.3389/fendo.2015.00051

22. Fletcher RB, Prasol MS, Estrada J, et al. p63 regulates olfactory stem cell self-renewal and differentiation. Neuron. 2011;72(5):748-759. doi:10.1016/j.neuron.2011.09.009

23. Fletcher RB, Das D, Gadye L, et al. Deconstructing olfactory stem cell trajectories at single-cell resolution. Cell Stem Cell. 2017;20(6):817-830 e818. doi:10.1016/j.stem.2017.04.003

24. Herrick DB, Lin B, Peterson J, Schnittke N, Schwob JE. Notch1 maintains dormancy of olfactory horizontal basal cells, a reserve neural stem cell. Proc Natl Acad Sci U S A. 2017;114(28):E5589-E5598. doi:10.1073/pnas.1701333114

25. Williams SK, Franklin RJ, Barnett SC. Response of olfactory ensheathing cells to the degeneration and regeneration of the peripheral olfactory system and the involvement of the neuregulins. J Comp Neurol. 2004;470(1):50-62. doi:10.1002/cne.11045

26. Barraud P, Seferiadis AA, Tyson LD, et al. Neural crest origin of olfactory ensheathing glia. Proc Natl Acad Sci U S A. 2010;107(49):21040-21045. doi:10.1073/pnas.1012248107

27. Ekberg JA, Amaya D, Mackay-Sim A, St John JA. The migration of olfactory ensheathing cells during development and regeneration. Neurosignals. 2012;20(3):147-158. doi:10.1159/000330895

28. Cao L, Su Z, Zhou Q, et al. Glial cell line-derived neurotrophic factor promotes olfactory ensheathing cells migration. Glia. 2006;54(6):536-544. doi:10.1002/glia.20403

29. Windus LCE, Chehrehasa F, Lineburg KE, et al. Stimulation of olfactory ensheathing cell motility enhances olfactory axon growth. Cell Mol Life Sci. 2011;68(19):3233-3247. doi:10.1007/s00018-011-0630-9

30. Vukovic J, Ruitenberg MJ, Roet K, et al. The glycoprotein fibulin-3 regulates morphology and motility of olfactory ensheathing cells in vitro. Glia. 2009;57(4):424-443. doi:10.1002/glia.20771

31. Nazareth L, Lineburg KE, Chuah MI, et al. Olfactory ensheathing cells are the main phagocytic cells that remove axon debris during early development of the olfactory system. J Comp Neurol. 2015;523(3):479-494. doi:10.1002/cne.23694

32. Su Z, Chen J, Qiu Y, et al. Olfactory ensheathing cells: the primary innate immunocytes in the olfactory pathway to engulf apoptotic olfactory nerve debris. Glia. 2013;61(4):490-503. doi:10.1002/glia.22450

33. Li Y, Zou T, Xue L, Yin ZQ, Huo S, Xu H. TGF-betal enhances phagocytic removal of neuron debris and neuronal survival by olfactory ensheathing cells via integrin/MFG-E8 signaling pathway. Mol Cell Neurosci. 2017;85:45-56. doi:10.1016/j.mcn.2017.08.006 
34. Wei Y, Miao X, Xian M, et al. Effects of transplanting olfactory ensheathing cells on recovery of olfactory epithelium after olfactory nerve transection in rats. Med Sci Monit. 2008;14(10):BR198-BR204.

35. Yue Y, Xue Q, Yang J, et al. Wnt-activated olfactory ensheathing cells stimulate neural stem cell proliferation and neuronal differentiation. Brain Res. 2020;1735:146726. doi:10.1016/j.brainres.2020.146726

36. Bao X, Xu X, Wu Q, et al. Sphingosine 1-phosphate promotes the proliferation of olfactory ensheathing cells through YAP signaling and participates in the formation of olfactory nerve layer. Glia. 2020;68(9):1757-1774. doi:10.1002/glia.23803

37. Sarnat HB, Flores-Sarnat L. Olfactory development, part 2: neuroanatomic maturation and dysgeneses. J Child Neurol. 2017;32(6):579-593. doi: $10.1177 / 0883073816685192$

38. Huart C, Rombaux P, Hummel T. Plasticity of the human olfactory system: the olfactory bulb. Molecules. 2013;18(9):11586-11600. doi:10.3390/ molecules 180911586

39. Kanaki K, Sato K, Kashiwayanagi M. Functional synapse formation between rat olfactory receptor neurons and olfactory bulb neurons in vitro. Neurosci Lett. 2000;285:76-78. doi:10.1016/S0304-3940(00)01028-4

40. Marei HE, Althani A, Afifi N, et al. Over-expression of hNGF in adult human olfactory bulb neural stem cells promotes cell growth and oligodendrocytic differentiation. PLoS One. 2013;8(12):e82206. doi:10.1371/journal.pone.0082206

41. Frontera JL, Cervino AS, Jungblut LD, Paz DA. Brain-derived neurotrophic factor (BDNF) expression in normal and regenerating olfactory epithelium of Xenopus laevis. Ann Anat. 2015;198:41-48. doi:10.1016/j.aanat.2014.10.010

42. Uranagase A, Katsunuma S, Doi K, Nibu K. BDNF expression in olfactory bulb and epithelium during regeneration of olfactory epithelium. Neurosci Lett. 2012;516(1):45-49. doi:10.1016/j.neulet.2012.03.051

43. Nieto-Estevez V, Defterali C, Vicario C. Distinct effects of BDNF and NT-3 on the dendrites and presynaptic boutons of developing olfactory bulb GABAergic interneurons in vitro. Cell Mol Neurobiol. 2021. doi:10.1007/s10571-020-01030-x

44. Badurek S, Griguoli M, Asif-Malik A, et al. Immature dentate granule cells require Ntrk2/Trkb for the formation of functional hippocampal circuitry. iScience. 2020;23(5):101078. doi:10.1016/j.isci.2020.101078

45. Wang W, Lu S, Li T, et al. Inducible activation of ERK5 MAP kinase enhances adult neurogenesis in the olfactory bulb and improves olfactory function. $J$ Neurosci. 2015;35(20):7833-7849. doi:10.1523/JNEUROSCI.3745-14.2015

46. Racekova E, Orendacova J. An olfactory bulbectomy model to study plasticity of the nervous system in rat: a review focused on published data from the Slovak Republic. Int J Neurosci. 2007;117(8):1067-1090. doi:10.1080/00207450600912271

47. Weng P-L, Vinjamuri M, Ovitt CE. Ascl3 transcription factor marks a distinct progenitor lineage for non-neuronal support cells in the olfactory epithelium. Sci Rep. 2016;6:38199. doi:10.1038/srep38199

48. Wang Y, Huang ZH. Morphological phenotypes of olfactory ensheathing cells display different migratory responses upon Slit-2. Exp Cell Res. 2012;318(15):1889-1900. doi:10.1016/j.yexcr.2012.05.024

49. Reginensi D, Carulla P, Nocentini S, et al. Increased migration of olfactory ensheathing cells secreting the Nogo receptor ectodomain over inhibitory substrates and lesioned spinal cord. Cell Mol Life Sci. 2015;72(14):2719-2737. doi:10.1007/s00018-015-1869-3

50. Oleszkiewicz A, Hanf S, Whitcroft KL, Haehner A, Hummel T. Examination of olfactory training effectiveness in relation to its complexity and the cause of olfactory loss. Laryngoscope. 2018;128(7):1518-1522. doi:10.1002/lary.26985

51. Altundag A, Cayonu M, Kayabasoglu G, et al. Modified olfactory training in patients with postinfectious olfactory loss. Laryngoscope. 2015;125 (8):1763-1766. doi:10.1002/lary.25245

52. Qiao X-F, Bai Y-H, Wang G-P, Li X, Zheng W. Clinical effects of two combinations of olfactory agents on olfactory dysfunction after upper respiratory tract infection during olfactory training. Rev Assoc Med Bras. 2020;66(1):18-24. doi:10.1590/1806-9282.66.1.18

53. Konstantinidis I, Tsakiropoulou E, Constantinidis J. Long term effects of olfactory training in patients with post-infectious olfactory loss. Rhinology. 2016;54(2):170-175. doi:10.4193/Rhino15.264

54. Geißler K, Reimann H, Gudziol H, Bitter T, Guntinas-Lichius O. Olfactory training for patients with olfactory loss after upper respiratory tract infections. Eur Arch Otorhinolaryngol. 2014;271(6):1557-1562. doi:10.1007/s00405-013-2747-y

55. Poletti SC, Michel E, Hummel T. Olfactory training using heavy and light weight molecule odors. Perception. 2017;46(3-4):343-351. doi:10.1177/ 0301006616672881

56. Damm M, Pikart LK, Reimann H, et al. Olfactory training is helpful in postinfectious olfactory loss: a randomized, controlled, multicenter study. Laryngoscope. 2014;124(4):826-831. doi:10.1002/lary.24340

57. Oleszkiewicz A, Bottesi L, Pieniak M, et al. Olfactory training with aromastics: olfactory and cognitive effects. Eur Arch Otorhinolaryngol. 2022. doi:10.1007/s00405-021-06810-9

58. Saatci O, Altundag A, Duz OA, Hummel T. Olfactory training ball improves adherence and olfactory outcomes in post-infectious olfactory dysfunction. Eur Arch Otorhinolaryngol. 2020;277(7):2125-2132. doi:10.1007/s00405-020-05939-3

59. Mori E, Petters W, Schriever VA, Valder C, Hummel T. Exposure to odours improves olfactory function in healthy children. Rhinology. 2015;53 (3):221-226. doi:10.4193/Rhino14.192

60. Mahmut MK, Pieniak M, Resler K, Schriever VA, Haehner A, Oleszkiewicz A. Olfactory training in 8-year-olds increases odour identification ability: a preliminary study. Eur J Pediatr. 2021;180(7):2049-2053. doi:10.1007/s00431-021-03970-y

61. Lamira JM, Soler ZM, Schlosser RJ. A pilot study of olfactory training in older hyposmic adults. Am J Rhinol Allergy. 2019;33(6):650-656. doi:10.1177/1945892419858793

62. Birte-Antina W, Ilona C, Antje H, Thomas H. Olfactory training with older people. Int J Geriatr Psychiatry. 2018;33(1):212-220. doi:10.1002/ gps. 4725

63. Konstantinidis I, Tsakiropoulou E, Bekiaridou P, Kazantzidou C, Constantinidis J. Use of olfactory training in post-traumatic and postinfectious olfactory dysfunction. Laryngoscope. 2013;123(12):E85-90. doi:10.1002/lary.24390

64. Kattar N, Do TM, Unis GD, Migneron MR, Thomas AJ, McCoul ED. Olfactory training for postviral olfactory dysfunction: systematic review and meta-analysis. Otolaryngol Head Neck Surg. 2021;164(2):244-254. doi:10.1177/0194599820943550

65. Choi BY, Jeong H, Noh H, Park JY, Cho JH, Kim JK. Effects of olfactory training in patients with postinfectious olfactory dysfunction. Clin Exp Otorhinolaryngol. 2021;14(1):88-92. doi:10.21053/ceo.2020.00143

66. Huang T, Wei Y, Wu D. Effects of olfactory training on posttraumatic olfactory dysfunction: a systematic review and meta-analysis. Int Forum Allergy Rhinol. 2021;11(7):1102-1112. doi:10.1002/alr.22758 
67. Jiang R-S, Twu C-W, Liang K-L. The effect of olfactory training on odor identification in patients with traumatic anosmia. Int Forum Allergy Rhinol. 2019;9(11):1244-1251. doi:10.1002/alr.22409

68. Haehner A, Tosch C, Wolz M, et al. Olfactory training in patients with Parkinson's disease. PLoS One. 2013;8(4):e61680. doi:10.1371/journal. pone. 0061680

69. Hummel T, Stupka G, Haehner A, Poletti SC. Olfactory training changes electrophysiological responses at the level of the olfactory epithelium. Rhinology. 2018;56(4):330-335. doi:10.4193/Rhin17.163

70. Kim BY, Park JY, Kim EJ, Kim BG, Kim SW, Kim SW. The neuroplastic effect of olfactory training to the recovery of olfactory system in mouse model. Int Forum Allergy Rhinol. 2019;9(7):715-723. doi:10.1002/alr.22320

71. Franceschini IA, Barnett SC. Low-affinity NGF-receptor and E-N-CAM expression define two types of olfactory nerve ensheathing cells that share a common lineage. Dev Biol. 1996;173(1):327-343. doi:10.1006/dbio.1996.0027

72. Barnett SC, Riddell JS. Olfactory ensheathing cells (OECs) and the treatment of CNS injury: advantages and possible caveats. $J$ Anat. 2004;204 (1):57-67. doi:10.1111/j.1469-7580.2004.00257.x

73. Kim BY, Park J, Kim E, Kim B. Olfactory ensheathing cells mediate neuroplastic mechanisms after olfactory training in mouse model. Am J Rhinol Allergy. 2020;34(2):217-229. doi:10.1177/1945892419885036

74. Marin C, Laxe S, Langdon C, et al. Olfactory training prevents olfactory dysfunction induced by bulbar excitotoxic lesions: role of neurogenesis and dopaminergic interneurons. Mol Neurobiol. 2019;56(12):8063-8075. doi:10.1007/s12035-019-1639-6

75. Hasegawa-Ishii S, Imamura F, Nagayama S, Murata M, Shimada A. Differential effects of nasal inflammation and odor deprivation on layerspecific degeneration of the mouse olfactory bulb. eNeuro. 2020;7(2):ENEURO.0403-19.2020. doi:10.1523/ENEURO.0403-19.2020

76. Veyseller B, Ozucer B, Aksoy F, et al. Reduced olfactory bulb volume and diminished olfactory function in total laryngectomy patients: a prospective longitudinal study. Am J Rhinol Allergy. 2012;26(3):191-193. doi:10.2500/ajra.2012.26.3768

77. Gürbüz D, Kesimli MC, Bilgili AM, Durmaz HÖ. Olfactory rehabilitation and olfactory bulb volume changes in patients after total laryngectomy: a prospective randomized study. Braz J Otorhinolaryngol. 2021. doi:10.1016/j.bjorl.2021.02.013

78. Negoias S, Pietsch K, Hummel T. Changes in olfactory bulb volume following lateralized olfactory training. Brain Imaging Behav. 2017;11(4):9981005. doi:10.1007/s11682-016-9567-9

79. Pellegrino R, Han P, Reither N, Hummel T. Effectiveness of olfactory training on different severities of posttraumatic loss of smell. Laryngoscope. 2019;129(8):1737-1743. doi:10.1002/lary.27832

80. Hosseini K, Zare-Sadeghi A, Sadigh-Eteghad S, Mirsalehi M, Khezerloo D. Effects of olfactory training on resting-state effective connectivity in patients with posttraumatic olfactory dysfunction. Acta Neurobiol Exp. 2020;80(4):381-388. doi:10.21307/ane-2020-035

81. Rezaeyan A, Asadi S, Kamrava SK, Khoei S, Zare-Sadeghi A. Reorganizing brain structure through olfactory training in post-traumatic smell impairment: an MRI study. J Neuroradiol. 2021. doi:10.1016/j.neurad.2021.04.035

82. Han P, Musch M, Abolmaali N, Hummel T. Improved odor identification ability and increased regional gray matter volume after olfactory training in patients with idiopathic olfactory loss. I-Perception. 2021;12(2):20416695211005811. doi:10.1177/20416695211005811

83. Al Ain S, Poupon D, Hetu S, Mercier N, Steffener J, Frasnelli J. Smell training improves olfactory function and alters brain structure. Neuroimage. 2019;189:45-54. doi:10.1016/j.neuroimage.2019.01.008

84. Gellrich J, Han P, Manesse C, et al. Brain volume changes in hyposmic patients before and after olfactory training. Laryngoscope. 2018;128 (7):1531-1536. doi:10.1002/lary.27045

85. Kollndorfer K, Fischmeister FP, Kowalczyk K, et al. Olfactory training induces changes in regional functional connectivity in patients with longterm smell loss. Neuroimage Clin. 2015;9:401-410. doi:10.1016/j.nicl.2015.09.004

86. Kollndorfer K, Kowalczyk K, Hoche E, et al. Recovery of olfactory function induces neuroplasticity effects in patients with smell loss. Neural Plast. 2014;2014:140419. doi:10.1155/2014/140419

87. Jiramongkolchai P, Jones MS, Peterson A, et al. Association of olfactory training with neural connectivity in adults with postviral olfactory dysfunction. JAMA Otolaryngol Head Neck Surg. 2021;147(6):502-509. doi:10.1001/jamaoto.2021.0086

Therapeutics and Clinical Risk Management

Dovepress

\section{Publish your work in this journal}

Therapeutics and Clinical Risk Management is an international, peer-reviewed journal of clinical therapeutics and risk management, focusing on concise rapid reporting of clinical studies in all therapeutic areas, outcomes, safety, and programs for the effective, safe, and sustained use of medicines. This journal is indexed on PubMed Central, CAS, EMBase, Scopus and the Elsevier Bibliographic databases. The manuscript management system is completely online and includes a very quick and fair peer-review system, which is all easy to use. Visit http://www. dovepress.com/testimonials.php to read real quotes from published authors.

Submit your manuscript here: https://www.dovepress.com/therapeutics-and-clinical-risk-management-journal 